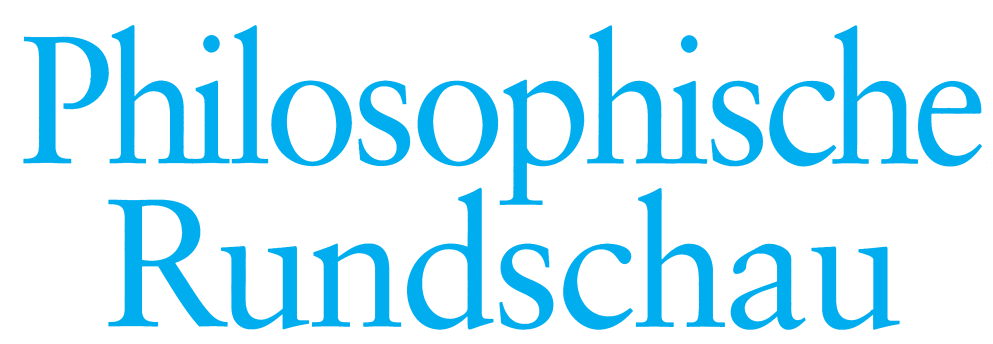

Eine Zeitschrift für philosophische Kritik

Band $\mathbf{6 0}$ Herausgegeben von

Heft 3 Jens Halfwassen - Pirmin Stekeler-Weithofer 2013 Bernhard Waldenfels

Helmuth Vetter »Es darf keine Heidegger-Scholastik aufkommen". Heidegger im Licht neuerer Literatur 179-206

Volker Schürmann Logos und Ethos der Leiblichkeit am Beispiel phänomenologischer Auskünfte 207-224

Henny Blomme Kants Raumbegriff in der Diskussion 225-240

Buchnotizen

Vittorio Hösle: Eine kurze Geschichte der deutschen Philosophie. Rückblick auf den deutschen Geist (Pirmin Stekeler-Weithofer) 241-242

Ulrich Sieg: Geist und Gewalt. Deutsche Philosophen zwischen Kaiserreich und Nationalsozialismus (Andreas Urs Sommer) 242-248

Denis Diderot: Philosophische Schriften (Martin Gessmann) 248-252

Kay Herrmann: Apriori im Wandel. Für und wider eine kritische Metaphysik der Natur (Andreas Brandt) 253-256

Hinweis

Georges Canguilhem: Schriften zur Medizin.

(Bernhard Waldenfels) 257 


\section{Philosophische Rundschau}

Eine Zeitschrift für philosophische Kritik

Redaktion:

Prof. Dr. Martin Gessmann, Hfg-Offenbach am Main, Schloßstr. 31, 63065 Offenbach/ Main, Telefon:069 / 80059-164, e-mail: Gessmann@hfg-offenbach.de

Die Philosophische Rundschau veröffentlicht ausschließlich deutschsprachige Artikel, die in der Regel vorher mit der Redaktion abgesprochen werden.

Mit der Annahme zur Veröffentlichung überträgt der Autor dem Verlag das ausschließliche Verlagsrecht für die Publikation in gedruckter und elektronischer Form. Weitere Informationen dazu und zu den beim Autor verbleibenden Rechten finden Sie unter www.mohr.de/phr.

Ohne Erlaubnis des Verlags ist eine Vervielfältigung oder Verbreitung der ganzen Zeitschrift oder von Teilen daraus in gedruckter oder elektronischer Form nicht gestattet. Bitte wenden Sie sich an rights@mohr.de.

Im Abonnement für Institutionen und Privatpersonen ist der freie Zugang zum Online-Volltext enthalten. Institutionen mit mehr als 20.000 Nutzern bitten wir um Einholung eines Preisangebots direkt beim Verlag. Kontakt: sandra.witt@mohr.de. Um den Online-Zugang für Institutionen / Bibliotheken einzurichten, gehen Sie bitte zur Seite: www.in gentaconnect.com/register/institutional. Um den Online-Zugang für Privatpersonen einzurichten, gehen Sie bitte zur Seite: www.ingentaconnect.com/register/personal.

(C) 2013 Mohr Siebeck GmbH \& Co.KG, Tübingen. - Die Zeitschrift und alle in ihr enthaltenen einzelnen Beiträge und Abbildungen sind urheberrechtlich geschützt. Jede Verwertung außerhalb der engen Grenzen des Urheberrechtsgesetzes ist ohne Zustimmung des Verlags unzulässig und strafbar. Das gilt insbesondere für Vervielfältigungen, Übersetzungen, Mikroverfilmungen und die Einspeicherung und Verarbeitung in elektronischen Systemen.

Satz und Druck: Gulde-Druck, Tübingen; Bindung: Nädele, Nehren.

ISSN 0031-8159 


\title{
Kants Raumbegriff in der Diskussion
}

\author{
Henny Blomme
}

Patrick Unruh: Transzendentale Ästhetik des Raumes. Zu Immanuel Kants Raumkonzeption. Würzburg 2007. Königshausen \& Neumann. 359 S.

Kiyoshi Chiba: Kants Ontologie der raumzeitlichen Wirklichkeit. Versuch einer anti-realistischen Interpretation der Kritik der reinen Vernunft, Berlin 2012. Walter De Gruyter. 427 S.

Oliver Schliemann: Die Axiome der Anschauung in Kants >Kritik der reinen Vernunftr. Berlin/New York 2010. Walter de Gruyter. 154 S.

Wo steht die Diskussion um den Kantischen Raumbegriff heute? Das Problem beginnt ganz offenbar schon bei der Erwähnung des Raumes in der Einzahl im Zusammenhang mit einer Form möglicher Aktualität. Schon im Verlaufe des 19. Jh.s war die Analytik der ästhetischen Urteilskraft, die Kant in seiner dritten Kritik formuliert hatte, weit wirkungsmächtiger als die transzendentale Ästhetik, in der er sein Raumkonzept entwickelte. Die von Kant in der Kritik der reinen Vernunft entwickelte Raumtheorie wurde von späteren Philosophen als nicht flexibel genug empfunden, um sich der veränderten Wirklichkeitsauffassung anzupassen - besonders dann, wenn es galt, durch die Entwicklung einer Vielzahl ästhetischer und lebensweltlicher Konzepte und der ihnen zugehörigen Räume eine neue Lebenswirklichkeit philosophisch zu erschließen. Das $20 \mathrm{Jh}$. hat jene Emanzipation der Raumwirklichkeit noch einmal radikalisiert und schließlich auf erkenntnistheoretische Grundsatzfragen zurückübertragen, wie sie bspw. in leibzentrierten Varianten der Phänomenologie zu finden sind.

Gleichzeitig verlor der Raumbegriff in seinem singulären Auftreten bei Kant, aber auch auf wissenschaftstheoretischer Seite selbst an Kredit. Mit der Ausdifferenzierung der mathematisch-naturwissenschaftlichen Konzepte, besonders in der theoretischen Physik an der Wende zum 20. Jh., wurde angenommen, dass Kants Auffassung des Raumes nicht mehr haltbar war. Dabei wurde jedoch nicht immer beachtet, dass Kant zwischen einem metaphysischen, einem geometrischen und einem physischen Raum unterschieden hat.

Zuletzt hat die vielfältige Entwicklung der Kulturwissenschaften zu Beginn des 21. Jh.s zu einer neuen, postmodernen Auffassung von Raumphilosophie geführt, in der noch einmal jene erkenntnistheoretischen wie auch ästhetisch-praktischen Ausdifferenzierungen auf eine neue Grundlage gestellt werden. Demnach geht bspw. Stephan Günzel davon aus, dass zuletzt jede Form einer methodisch gestützten Codierung unserer Welt- 
wahrnehmung bereits zur Ausbildung eines eigenen Wirklichkeitsraums führt und damit einen höchst relativen Raumbegriff nahelegt.

Jene Entwicklung sei deshalb so summarisch anzitiert, weil keiner der folgenden Beiträge den Versuch macht, in dieser nachkantischen Raumdebatte an entscheidender Weichenstellung einzuhaken. Es handelt sich bei der gegenwärtigen Literatur zum Kantischen Raumbegriff vielmehr um Spezialuntersuchungen im Bereich der Geschichte der Philosophie. Sie versuchen also nicht, Kants Raumtheorie mit den sich aktuell in Umlauf befindenden Philosophien über den Raum zu verknüpfen.

In dieser Diskussionslinie sollen drei Studien vorgestellt werden. Zum einen geht es um den kantimmanenten Aufweis, dass die Kritik der späteren Mathematiker und Physiker an Kants notwendiger Einheitsvorstellung des Raumes Kant in Wahrheit nicht trifft; zum anderen wird Kant in die analytische Debatte um Realismus und Anti-Realismus involviert, dies unter der Voraussetzung, dass dabei immer noch der Cartesische Dualismus von Ich und Welt gilt und Kant demnach als Transzendentalphilosoph noch nicht in dem ganzen Umfange, den die Kopernikanische Wende mit sich bringt, ernst genommen werden muss. Eine kantphilologische Arbeit bildet den Abschluß. In ihr geht es um die ungeschriebenen `Axiome der Anschauung< Kants und die Frage, wie diese konsistent gedacht und schlüssig ausformuliert werden können.

\section{$I$.}

Warum fängt die Kritik der reinen Vernunft mit einer transzendentalen Ästhetik an? Forscher wie Michael Friedman, Jaakko Hintikka, Darius Koriako und Charles Parsons haben die Bedeutung dieses ersten Teiles der transzendentalen Elementarlehre vor allem darin gesehen, dass Kant meinte, mit der in ihr vorgestellten Raum- und Zeitauffassung eine glaubwürdige Begründung der Apodiktizität mathematischer Erkenntnisse liefern zu können. Angesichts der zu Kants Lebzeiten unvorhersehbaren Entwicklung, die die Mathematik seit Anfang des 19. Jahrhunderts durchgemacht hat - wobei zum Beispiel die Geometrie nicht mehr nur euklidische Geometrie sein wird -, stellt sich dann aber unvermeidlich die Frage, ob die kantische Ästhetik eine solche grundlegende Funktion auch im nachkantischen Zeitalter behalten kann. Jedes Mal, wenn diese Frage eine verneinende Antwort erhält, bietet sich die Gelegenheit, sich mit einer gewissen Erleichterung von der hochparadoxen kantischen Raum- und Zeitlehre zu verabschieden, und die transzendentale Ästhetik wird dann auch von vielen Forschern gerne als der am wenigsten gelungene Teil (wenn nicht sogar als vorkritischer Rest innerhalb) der ersten Kritik angesehen. 
Patrick Unruh geht es in seiner hier zu besprechenden Studie dagegen darum, zu zeigen, wie Kants transzendentale Ästhetik als eigenständige philosophische Untersuchung gesehen werden kann und innerhalb des gesamten kritischen Projekts auch noch eine neue Antwort bietet auf traditionelle Fragen bezüglich der Natur des Raumes. Die Untersuchung der Sinnlichkeit wird von Kant demnach nicht mit dem Zweck geführt, die Geometrie als Wissenschaft zu begründen, sondern um die apriorischen sinnlichen Elemente der Erscheinung aufzuweisen. Zugleich findet Kant mit dieser Untersuchung einen Ausweg aus der philosophischen Sackgasse, in welche die Diskussion zwischen Newton und Leibniz in Bezug auf die Natur des Raumes geführt hatte. Dementsprechend beginnt Unruh das erste Kapitel seines Buches mit einer bündigen Besprechung der Newtonschen und Leibnizschen Raumkonzeptionen, um anschließend zu zeigen, wie sich Kants Raumauffassung in der Auseinandersetzung mit diesen beiden großen, sich bekämpfenden Raumtheorien seiner Zeit ausgebildet hat.

In der Leibniz-Wolffschen Schule gilt der Raum in Kants Rückblick als ein lediglich von der Erfahrung abstrahierter und durch den Verstand verworren vorgestellter Inbegriff der Verhältnisse von Dingen. Weil der Unterschied zwischen deutlichen und verworrenen Vorstellungen ein rein logischer ist, verkenne diese Schule die fundamentale Eigenständigkeit der Sinnlichkeit. Stattdessen intellektualisiere sie die Sinnlichkeit und bestimme sie als "mangelhaften" Verstand. Die Entdeckung, dass der Raum eine grundsätzlich vom Verstand unabhängige Natur hat, ist demnach wesentlich für Kants Abschied von der rationalistischen Raumlehre. Kant beschreibt diese Entdeckung zum ersten Male in seinem Text Von dem ersten Grunde des Unterschiedes der Gegenden im Raume (1768). Dass der Raum eine eigenständige Natur hat, beweist Kant dort anhand einiger Überlegungen zu sogenannten inkongruenten Gegenstücken. Inkongruente Gegenstücke sind Gegenstände, die sich, obwohl sie sich in allen messbaren Eigenschaften ähnlich sind, doch nicht in einer selben Raumform einschließen lassen. Auch wenn man von allen messbaren Unterschieden zwischen meiner rechten und meiner linken Hand abstrahiert (und also unterstellt, dass es zwischen ihnen keine messbare Unterschiede, wie einen längeren oder dickeren Daumen usw. gibt), kann die rechte Hand nicht im selben Raum eingeschlossen werden wie die linke. In der Geometrie kann man inkongruente Gegenstücke konstruieren, wie zum Beispiel inkongruente sphärische Dreiecke. Da die begriffliche Beschreibung von überall ähnlich gedachten, doch letztlich inkongruenten Händen (oder von überall ähnlichen aber inkongruenten sphärischen Dreiecken) völlig identisch ist, muss ihr Unterschied auf einem anderen Verhältnis zum Raum beruhen. Die "Richtung» oder "Orientiertheit» (»Linksheit» oder »Rechtsheit») ist demnach keine Eigenschaft, die einem Ding als solchem anhängt, sondern eine, die nur im Verhältnis des Dinges zu einem als »absolut" zu denkenden Raum zum Vorschein kommt.

Es ist aber philosophisch problematisch, den Raum wie Newton als absolutes Behältnis der Dinge zu konzipieren. Erstens wäre der Raum dann ewig und unendlich wirklich, auch wenn sich gar keine wirklichen Dinge in ihm befänden. Ein solcher Begriff vom Raum ist widersprüchlich, weil der Raum dann zugleich als Form und Materie gelten müsste. Zweitens müsste der Raum, wenn er als 
wirkliche Bedingung alles Wirklichen existierte, nach Kant (der darin Leibniz folgt) sogar die Bedingung des Daseins Gottes sein. Wenn aber die von Kant entdeckte "Absolutheit" des Raumes später kombiniert wird mit ihrer transzendentalen Idealität, hat er auch diese Probleme überwunden: da der Raum die bloß subjektive Form der äußeren Anschauung ist, kann er einerseits im oben umschriebenen Sinne absolut bleiben und muss andererseits nicht als in sich widersprüchlicher Begriff einer ewigen "materiellen Form« konzipiert werden.

Obwohl die Entdeckung der philosophischen Bedeutung von inkongruenten Gegenstücken nach Kant als Gnadenstoß für die rationalistische Raumlehre gilt, kann er feststellen, dass sein kritisches Raumverständnis auch ein anderes Problem dieser Raumlehre behebt, nämlich die Anwendbarkeit der Mathematik. Dort, wo Newton und seine Anhänger die apriorische Gültigkeit der Geometrie zwanglos erklären konnten (wenn sämtliche Dinge sich im Raum befinden, kann es nichts darüber hinaus geben, das nicht ihm und seinen Gesetzen gemäß wäre), konnten die Rationalisten die Anwendbarkeit der Mathematik nicht auf eine philosophisch befriedigende Weise erklären. Da nach Kant für letztere alle Eigenschaften des Raumes von kontingenter Erfahrung abstrahiert sind, kann die Wissenschaft vom Raum für sie eigentlich nur a posteriori statthaben. Als Folge kann die Mathematik nicht mehr apodiktisch gewiss auf die Erfahrung angewendet werden.

Kants Geniestreich im Bereich der theoretischen Philosophie war seine revolutionäre Ansicht, dass nicht nur der Verstand, sondern auch die Sinnlichkeit apriorische Vorstellungen gibt, die die Art der Gegebenheit der Gegenstände wesentlich bestimmen. Die transzendentale Ästhetik lehrt nun bekanntlich, dass die Untersuchung des Anschauungsvermögen der transzendentalen Subjektivität sich nur auf zwei apriorische Anschauungen richten muss: Raum und Zeit. Sie zeigt, dass alle Gegenstände, weil sie eine Art Vorstellung in uns sind, auch in der Zeit sind. Gegenstände des äußeren Sinnes sind zudem im Raum (und nur als räumliche Gegenstände sind sie wirkliche Gegenstände). Der Raum ist also nur deswegen Gegenstand der Transzendentalphilosophie, weil er als apriorische Vorstellung selbst notwendig ist, um dasjenige, was dem äußeren Sinn gegeben wird, als Gegenstand oder Gegenstände anschauen zu können. Weil die transzendentale Ästhetik zeigen muss, wie der Raum dies möglich macht, lehrt sie auch, was wir - philosophisch gesehen - a priori vom Raum (nun selbst als Gegenstand betrachtet) erkennen können. Unruh liefert hier nicht nur die nötigen Begriffserklärungen, um die Raumerörterungen verstehen zu können; er erklärt auch, warum es nach Kant nicht der Fall sein kann, dass wir vom Ding an sich affiziert werden - obwohl manche Interpreten nach Jacobi und auch nach dem bekannten Kantforscher Hans Vaihinger noch immer genau eine solche stranszendente Affektion bei Kant anzutreffen meinen (obwohl dieselben Interpreten nicht nachlassen, auf die systematische Inkohärenz einer solchen These aufmerksam zu machen). Die Folge von Kants Raum- und Zeitverständnis in der transzendentalen Ästhetik ist, dass alle Gegenstände, die von uns erkannt wer- 
den können (sowohl empirisch als a priori) keine Dinge an sich sein können. Es wäre nun problematisch, wie Unruh schreibt, wenn »[...] das Affizieren zu den Funktionen eines Dinges an sich gehören sollte, denn durch Affektion - als Funktion des Affizierenden - wird ja gerade, wie überhaupt durch jede Tätigkeit, ein Kontakt zu etwas anderem hergestellt, so dass das Ding gerade nicht mehr an sich betrachtet wird, sondern als im Verhältnis, d.h. nach einer solchen bedingten Bestimmung einer Sache, die nicht vorgestellt werden kann, wenn sie an sich selbst betrachtet wird." Das kausal Affizierende ist demnach vielmehr »einfach ein empirisches Ding« (S. 109-110).

Unruh erklärt auch sehr gut, wie das Argument der transzendentalen Erörterung des Raumes laufen soll. Ein Verständnis der transzendentalen Erörterung setzt voraus, dass man weiß, warum Kant meint, die Urteile in der Geometrie seien synthetisch a priori. Nachdem zurückgegriffen wurde auf die Einleitung der Kritik der reinen Vernunft, um die Grundbegriffe von Kants Urteilstheorie in Erinnerung zu rufen und die Erörterung präzise deuten zu können, bietet Unruh eine Auseinandersetzung mit der Literatur zu Kants Auffassung von Geometrie und mit der Frage, ob die nicht-euklidische Geometrie Kants transzendentale Ästhetik in irgendwelcher Weise ungültig machen könnte. Unter anderem Helmholtz, Husserl, Russell, Carnap und Strawson haben die von Kant behauptete synthetische Apriorität der geometrischen Urteile bestritten und ihm vorgeworfen, dass er nicht zwischen reiner (mathematischer) und angewandter (physikalischer) Geometrie unterscheidet. Die reine (mathematische) Geometrie wäre demnach a priori, weil sie analytisch sei, »[...] während die angewandte Geometrie zwar synthetisch sein könne, aber auch zu einem empirisch überprüfbaren Hypothesensystem über die Eigenschaften des physikalischen Raumes geworden sei und mithin nicht mehr apriorische Gültigkeit für sich in Anspruch nehmen könne.«(S. 259). Nach dieser Auffassung wäre dann aber auch wenigstens Kants transzendentale Erörterung des Raumes ungültig. Unruh zeigt darauf, dass das in dieser Unterscheidung benutzte Verständnis von Analytizität mit Kants Auffassung unvereinbar ist. Er kommt zu folgendem Ergebnis: "Zwar war es Immanuel Kants Überzeugung, dass die euklidische Geometrie allein gültig ist und dass der wissenschaftliche Raum euklidisch verfasst ist, doch qua Transzendentalphilosoph hat Kant zu solchen Überzeugungen meiner Ansicht nach nicht kommen können. Weil der transzendentale Idealismus sich [...] vor der Möglichkeit einander widersprechender mathematischer Theorien bewegt, kann die Entdeckung nichteuklidischer Geometrien in der Mathematik wie auch ihre Anwendbarkeit auf die Erfahrung für sie keine ruinöse Bedrohung bedeuten, und ein Irrtum Kants in diesem Be- 
reich kann keine grundsätzlichen Auswirkungen auf sein transzendentalphilosophisches Projekt haben." (S. 264).

Dieses Buch ist ein wahres Geschenk für die Kantforschung. Es ist das Beste, was bis jetzt in deutscher Sprache über die transzendentale Ästhetik vorgelegt wurde. Es hat keine nennenswerten Schwachstellen und zeugt auf jeder Seite von der souveränen Kompetenz seines Autors.

\section{II.}

Es gibt in der Kant-Forschung eine vor allem seit den neunziger Jahren wieder auflebende Debatte über die Frage, ob Kant als Realist oder Anti-Realist einzustufen sei. Der Untertitel von Kiyoshi Chibas Buch «Kants Ontologie der raumzeitlichen Wirklichkeit $\_$"Versuch einer anti-realistischen Interpretation der Kritik der reinen Vernunft« - sagt sogleich, was bevorsteht: ein Versuch, die kritische Philosophie exklusiv mit der philosophischen Position des Anti-Realismus zu verbinden.

Aber was genau soll die philosophische Position des Anti-Realismus besagen? Es gibt in der Geschichte der Philosophie viele Varianten von Anti-Realismus. Wie vor ihm schon Carl Posy und Lucy Allais (u. a.m.), will Chiba anhand der spezifisch von Michael Dummett beschriebenen Unterscheidung des Realismus und Anti-Realismus die kritische Philosophie interpretieren. Der Anti-Realismus im dummettschen Sinne besagt nun eigentlich nur, wie Chiba erklärt, dass Wahrheit in irgendeinem Sinne erkenntnisabhängig sei. Das ist eine sehr minimale Definition von Anti-Realismus, da sie vom Anti-Realisten nur fordert, dass er die Wahrheit von Aussagen in irgendeiner Weise mit der Verifizierbarkeit dieser Aussagen verbindet. Demnach behauptet dieser dummettsche Anti-Realismus nicht, dass es überhaupt keine erkenntnisunabhängige Entitäten (Chiba nennt als Beispiel die kantischen "Dinge an sich" - S. 43) oder Tatsachen gibt, oder, dass erkenntnis-unabhängiger Faktoren überhaupt keinen Einfluss auf die Wahrheit haben können. Wie Chiba schreibt, besteht der »[...] Unterschied zwischen Realismus und Anti-Realismus [im dummettschen Sinne (HB)] gerade darin, dass der letztere die Verifizierbarkeit für konstitutiv für die Wahrheit selbst hält. Der Anti-Realismus schließt daher die verifikationstranszendente Wahrheitskonzeption jeder Art begrifflich aus. Was den Anti-Realismus auszeichnet, ist diese enge Verknüpfung der Wahrheit (bzw. Wirklichkeit) mit epistemischen Momenten auf unserer Seite." (S. 25). In diesem Anti-Realismus geht es also um die Wahrheit von Aussagen, nicht um die Existenz von Gegenständen. Kant selbst bespricht nun, wie auch Chiba bemerkt, meistens nicht die Wahrheit der Aussagen über Gegenstände, sondern vielmehr die Existenz der Gegenstände in Raum und Zeit. Kant behauptet lediglich, dass raumzeitliche Gegenstände in gewisser Weise subjektabhängig sind. Um diese Subjektabhängigkeit präzise zu verstehen, sei es Chiba zufolge aber "[...] ratsamer, die Wahrheit der Aussagen zu analysieren als die Existenz der Gegenstände." (S. 26). Man könnte fragen, warum dies "ratsamer« ist. Weil nur so »die bikonditionale Darstellung einer realistischen oder anti-realistischen Position verfügbar" wird, sagt Chiba (ebd.). Eine bikonditionale Darstellung ist notwendig, damit man philosophische Positionen auf eindeutige Weise dem Realismus oder dem Anti-Realismus zuordnen kann. 
Diese Überlegung deutet darauf hin, dass man zur Beurteilung der Aufgabe, die Chiba sich in diesem Buch gestellt hat, zwei ganz unterschiedliche Fragen beantworten muss. Die erste lautet: Kann Chiba mittels seiner von der dummettschen Terminologie inspirierten Interpretation eine fruchtbare Behandlung von dem (nicht genuin kantischen) Problem bieten, ob der Kritik der reinen Vernunft zufolge die Wahrheit von Aussagen über die Existenz von raumzeitlichen Gegenständen abhängig oder nicht abhängig vom Subjekt (oder von den Subjekten) ist? Zu dieser Frage schreibt Chiba selbst folgendes:

"Meine anti-realistische Interpretation deutet den Gegensatz von TrR [ = transzendentaler Realismus] und TrI [= transzendentaler Idealismus] als Gegensatz um die Wahrheitskonzeption für raumzeitbezügliche Aussagen. Damit behaupte ich nicht, dass Kant seinen TrI wissentlich mit Rekurs auf die Wahrheitskonzeption ausgearbeitet hat. Die dummettsche Begrifflichkeit soll nur ein analytisches Rüstzeug sein, um Kants Ontologie der raumzeitlichen Wirklichkeit klar und präzise auszulegen. Sofern sie dieser Zielsetzung gerecht wird, ist die Anwendung der Begrifflichkeit der heutigen Philosophie sowie Logik auf die Kant-Interpretation kein Anachronismus. Ob sie dafür tatsächlich fruchtbringend ist oder nicht, ist allerdings nur post festum zu bewerten." (S. 44)

Nun, post festum muss man erkennen, dass Chiba in der Tat in der Lage ist, mit der Begrifflichkeit der heutigen Philosophie die von Kant vertretenen Lehren zu analysieren und manchmal zu erstaunlichen Einsichten zu gelangen. Dabei hilft ihm seine gute Vertrautheit mit einer großen Anzahl von Werken - sowohl aus der so genannten analytischen Philosophie als auch aus der Kant-Literatur - die für seine Studie auch nur irgendwie relevant sein könnten. Diese sich der analytischen Begrifflichkeit bedienenden Untersuchung kommt aber nicht $\mathrm{zu}$ einem eindeutigen Ergebnis: zwar ist Kant im dummettschen Sinne als "Anhänger« des Anti-Realismus zu betrachten, aber es bleibt unklar, welcher Variante davon Kant anhängt. Was die zeit-relative Variante betrifft ("Eine Aussage ist zum Zeitpunkt t wahr gdw. sie bis t verifiziert worden ist « - S. 274), ist es nach Chiba ausgemacht, dass diese Variante Kants Position nicht getreu wiedergibt. Chiba konstruiert dann insgesamt fünf Versionen des Anti-Realismus, die er alle "als mögliche Optionen für den kantischen TrI [...].« (S. 331) gelten lässt. Was aber »Kants Selbstverständnis anbelangt, so dürfte wohl ZN [zeit-neutrale Variante des Anti-Realismus] seiner Ansicht am nächsten stehen, denn er hat über die Probleme, die durch die Möglichkeit des Verlusts der Erkenntnisse sowie die Anfechtbarkeit empirischer Aussagen verursacht werden, kaum reflektiert [...]«(S. 331).

Die zweite Frage, die man sich zur Beurteilung dieses Buches stellen muss, lautet wie folgt: Zeigt Chiba in der Tat, dass die Anwendung der dummettschen Unterscheidung von Realismus und Anti-Realismus uns 
besser als andere methodische Verfahren in die Lage versetzt, zu verstehen, was Kant genau meint wenn er sagt, dass die Gegenstände (wie sie uns erscheinen) nicht unabhängig vom Subjekt gegeben sind?

Dabei muss man zunächst feststellen, dass diese Studie sich nicht auf das eigentliche Thema der Kritik der reinen Vernunft bezieht. Das Hauptanliegen der ersten Kritik ist bekanntlich eine Untersuchung der Möglichkeit einer apriorischen Erkenntnis vom Gegenstand überhaupt. Diese Untersuchung nennt Kant ganz am Anfang seines Buches eine "Kritik», und er bestimmt sie zugleich als den ersten und wichtigsten Teil eines "Systems der Transzendentalphilosophie». Was Kant "Kritik« nennt, würde man heute wahrscheinlich als »erkenntnistheoretische Untersuchung « bezeichnen. Es ist demnach keine metaphysische Untersuchung über Gegenstände als existierende Gegenstände. Andererseits ist es richtig, dass die Zeit- und Raumlehre der transzendentalen Ästhetik - als erster Teil der Kritik des apriorischen Erkenntnisvermögens - auch die Weise bedingt, in der wir von der Existenz von raumzeitlichen Gegenständen reden können: wir müssen nämlich die Existenz von raumzeitlichen Gegenständen als Dingen an sich radikal ablehnen. Da die Ästhetik beweist, dass Räumlichkeit und Zeitlichkeit keine möglichen Eigenschaften von Dingen an sich sind, und wir uns die Dinge immer nur als raumzeitliche Objekte vorstellen können, können die von uns vorgestellten Objekte keine Dinge an sich sein. Deswegen spricht Kant von der transzendentalen Idealität von Raum und Zeit. Dagegen kommt der Terminus "transzendentaler Idealismus" in der Kritik von 1781 zum ersten Mal im Abschnitt „Kritik des vierten Paralogism der transzendentalen Psychologie«(A369) vor. Dort tritt der transzendentale Idealismus im Rahmen einer Kritik der dogmatischen Metaphysik auf, weil diese nun gerade doch in einem metaphysischen Sinne über die Existenz von Gegenständen spricht. Der cartesianische Idealismus zum Beispiel, den Kant einen "problematischen" oder "skeptischen" Idealismus nennt, bezweifelt, ob wir als Ursachen von den uns gegebenen Wahrnehmungen existierende Objekten annehmen dürfen, weil die Ursache von einer gegebenen Wirkung immer qualitativ unbestimmt ist. Über die Existenz der Ursache einer Wahrnehmung können wir, ausgehend von dieser Wahrnehmung, nichts aussagen, weil die Ursache sowohl das wahrgenommene Objekt als das wahrnehmende Subjekt sein könnte (oder auch bei Descartes Gott oder ein malin génie). Weil bei Kant alle Vorstellungen von Objekten als Vorstellungen Gegenstand des inneren Sinnes sind, könnte man den Eindruck haben, dass sich auch bei ihm ein solches Problem stellt. Da aber der Raum - als Form der äußeren und also auch der äuBerlichen Objekte - selbst in uns ist, ist die Existenz von äußerlichen Objekten bei Kant nicht problematisch. Deswegen kann Kant von seinem nicht-metaphysischen transzendentalen Idealismus behaupten, dass dieser Idealismus im kritischen Verständnis zugleich einen empirischen Realismus impliziert.

Es scheint mir, dass Chibas Versuch, mit Hilfe von nicht-kantischen Begriffsbestimmungen die kantische »Subjektabhängigkeit" der Existenz der Gegenstände zu verstehen, darunter leidet, dass einige für ein solches Verständnis notwendige Begriffe und Lehrstücke aus der Kritik der reinen Vernunft nicht behandelt werden. Chiba behandelt zwar alle Stellen, an denen Kant etwas über den transzendentalen Idealismus in seinem Sinne sagt, also auch die Diskussion mit dem problematischen Idealismus. Seine 
Analysen sind zwar immer lehrreich, aber verfehlen meines Erachtens den folgenden, wichtigen Punkt. Dieser läßt darauf schließen, gemäß der Überlegungen, die Kant zu dem vom kritischen Philosoph einzunehmenden doppelten Standpunkt anstellt, dass bspw. die Tätigkeit aller empirischen Wissenschaftler in keiner Weise von der von Kant betonten transzendentalen Idealität der Erscheinungen betroffen ist. Die von Kant auch stark betonte empirische Realität der Erscheinungen sichert dabei den common sense Realismus, den alle nicht-Philosophen - wie auch der Philosoph in seinem weltlichen Leben - im Normalfall vertreten (und vertreten sollten).

Gerade weil Kants transzendentaler Idealismus ausschließt, dass die Existenz der Erscheinungen automatisch vielleicht auch die Existenz von diesen Erscheinungen als Dinge an sich impliziert, kann sich Kant relativ kurz halten in Bezug auf das, was die Existenz der den uns erscheinenden Gegenstände anbelangt: Es gibt kein apriorisches Kriterium, um über die Existenz von Gegenständen, die nicht Teil unserer Erfahrung wären, etwas auszusagen. In diesem Sinne ist auch das zweite Postulat des empirischen Denkens zu verstehen: wirklich ist was mit den materialen Bedingungen der Erfahrung (der Empfindung) zusammenhängt. (A218/B266). Da er aber meint, dass der materiale Grund der Empfindung auf eine transzendente Affektion (durch Dinge an sich) zurückzuführen ist, kann Chiba die kantische Lehre insgesamt als Anti-Realismus deuten. Die im vierten Paralogismus behauptete transzendentale Idealität aller Erscheinungen, die dort im Rahmen der Kritik an einem problematischen Idealismus gedeutet werden muss, soll nun aber gerade illustrieren, warum Dinge an sich lediglich problematische Gegenstände sein können. Wir können von Dingen an sich nichts wissen, also auch nicht, ob sie existieren oder nicht. Deswegen ist der materiale Grund der Empfindung in den uns (empirisch) affizierenden empirischen Gegenständen zu suchen. Die (notwendigerweise empirische) Wissenschaft, die die empirische Affektion untersuchen könnte, darf aber kein Teil sein der in der Kritik der reinen Vernunft vorgelegten Untersuchung des Erkenntnisvermögens, weil diese nur die notwendigen apriorischen Bestimmungen eines Gegenstandes überhaupt zum Thema hat.

In der Analyse von problematischen Fällen, z. B. hinsichtlich der Frage, ob es Menschen auf dem Mond gebe, knüpft Chiba nahtlos an das von Kant Behauptete an. Er stellt aber zu Recht auch fest, dass Kant die in der heutigen analytischen Philosophie besprochenen Probleme wie Anfechtbarkeit und Möglichkeit eines Verlusts von empirischer Erkenntnis nicht bespricht. Auch das dürfte aber damit zusammenhängen, dass solche erkenntnistheoretischen Fragen, die als Gedankenexperimente auf empirische Tatsachen gerichtet sind, (wenigstens nach Kant) nicht als wesentlicher Teil einer Untersuchung des apriorischen Erkenntnisvermögens betrachtet werden können. 
III.

Die Raumdiskussion bei Kant hat, wie eben gesehen, damit zu tun, dass die in Frage stehende transzendentale Raumvorstellung immer wieder an der Schnittstelle metaphysischer Analysen, mathematisch-geometrischer Gewissheiten und empirischer Evidenzen operiert. Fraglich ist dabei grundsätzlich und erstens, wie sich die Notwendigkeit von synthetischen Urteilen a priori (nicht nur in der Mathematik) auf den Raum als subjektive Form der Anschauung gründen kann; und wie sich zweitens sowohl die apriorische Raumvorstellung aus der transzendentalen Ästhetik als auch der geometrische Raum mit der Anschauung der empirischen Dinge als räumlichen Dingen verbinden lassen: eine apriorische Ordnungsleistung der Sphären Raum (und Zeit) muss mit der Möglichkeit empirischer Anschauung und empirisch verifizierbarer Urteile zusammen gedacht werden.

Oliver Schliemann behandelt in seiner Studie über die >Axiome der Anschauung in Kants >Kritik der reinen Vernunft« einen Aspekt dieser von Kant im Grundsatzkapitel dargelegten Möglichkeit. Es handelt sich also um einen Beitrag zur Kantphilologie, in dem jedoch konzeptionelle Grundlagen geklärt werden, die für die Raumdiskussion wichtig sind.

Folgendes ist das philologische Ausgangsproblem: Obwohl Kant schreibt, dass die Tafel mit den zwölf Kategorien die "ganz natürliche Anweisung zur Tafel der Grundsätze«(A161/B200) gibt, findet man in der Kritik der reinen Vernunft keine zwölf Grundsätze. In den beiden ersten Abschnitten des Grundsatzkapitels (»Die Axiome der Anschauung" und »Die Antizipationen der Wahrnehmung«) gibt Kant lediglich das »Prinzip« dergleichen Axiome oder Antizipationen (B201,207). Der wackere Leser stellt sich dann auch bald die Frage, welche Grundsätze nun mit diesen Axiomen und Antizipationen gemeint sein können, und ob es davon auch (wie bei den Analogien und Postulaten) jeweils drei oder sogar mehrere gibt.

Oliver Schliemann stellt sich diese Frage mit Bezug auf die Axiome. Wie der Autor in der Einleitung bemerkt, liegt es nahe, zu unterstellen, dass es sich bei den von Kant genannten Axiomen der Anschauung um Axiome der Mathematik (insbesondere der Geometrie) handelt. Schon George Samuel Albert Mellin schrieb in seinem Ende des 18. Jahrhunderts erschienenen Encyclopädischen Wörterbuch der kritischen Philosophie, dass Kant mit den Axiomen der Anschauung die "wahren Axiomen [...], nehmlich die Axiomen der Mathematik« (Bd.1, S. 452) gemeint hat, und neuerdings wurde diese Ansicht auch vertreten von Paul Guyer, Jay Rosenberg und Holm Tetens. Es wird dabei aber nie gesagt, wie diese Axiome der Mathematik (oder der Geometrie und eventuell sogar der Chronometrie) genau lauten. Deswegen hat Schliemann sich vorgenommen, den exakten Wort- 
laut der von Kant genannten, aber nicht explizit vorgestellten Axiome der Anschauung zu rekonstruieren. Das ist eine hoch ambitionierte Aufgabe, und man muss loben, dass Schliemann in seinem Eifer nicht ruht, bis ein eindeutiges Ergebnis vorliegt.

Wesentlich für Schliemanns Rekonstruktionsversuch ist Kants in den Prolegomena gegebene Auslegung der Grundsätze als "[...] Sätze, welche alle Wahrnehmung (gemäß gewissen allgemeinen Bedingungen der Anschauung) unter [...] reine Verstandesbegriffe subsumieren." (AA IV 302) Mit den reinen Verstandesbegriffen sind hier natürlich die Kategorien gemeint. Demgemäß subsumieren die Grundsätze alle Wahrnehmung unter Kategorien. Da nun Kant vom transzendentalen Schema sagt, dass es die Subsumtion der Erscheinungen unter die Kategorien vermittelt (Cf. A139/ B176), und dass das Schema die formale Bedingung der Urteilskraft ist, unter der etwas in der Anschauung gegeben sein kann (Cf. A247/B304), identifiziert Schliemann die "gewissen allgemeinen Bedingungen der Anschauung" (gemäß welchen die Grundsätze die Wahrnehmung unter Kategorien subsumieren) mit den Schemata der reinen Verstandesbegriffe. Die allgemeine Struktur eines Grundsatzes des reinen Verstandes sollte demnach so lauten: Jede Erscheinung, die die Bedingung Y erfüllt, ist ein $\mathrm{X}$ «, wobei $\mathrm{X}$ eine Kategorie ist und $\mathrm{Y}$ das Schema dieser Kategorie.

Um die den drei Kategorien der Quantität korrespondierenden Grundsätze (wovon Schliemann also vermutet, dass Kant sie sehr wohl vor $\mathrm{Au}-$ gen hatte, aber der Kürze wegen nicht niedergeschrieben hat) nachträglich formulieren zu können, muss man demnach zuerst wissen, welche Schemata den drei Kategorien der Quantität zugeordnet werden müssen. Damit verdoppelt sich aber Schliemanns Problem einer Rekonstruktion der Axiome: Kant spricht im Schematismuskapitel lediglich von einem "Schema der Größe« (A142/B182) und macht von den gesuchten Schemata der einzelnen Quantitätskategorien (Einheit, Vielheit, Allheit) keine weitere Anzeige - also müssen jetzt auch diese Schemata rekonstruiert werden.

Als wichtiger Hinweis für die Rekonstruktion der Schemata der einzelnen Quantitätskategorien betrachtet Schliemann die Art und Weise, wie Kant den Unterschied zwischen Erscheinung und Phänomen einführt. Auf Seite 52 bemerkt Schliemann dazu, dass 'Erscheinungen (als unbestimmte Gegenstände der empirischen Anschauung) nur `Phänomener genannt werden können, "so fern sie als Gegenstände nach der Einheit der Kategorien gedacht werden" (A248), und sagt darauf folgendes: »diejenige Kategorie, nach deren Einheit ein solcher Gegenstand unumgänglich gedacht werden muss, [ist] die Kategorie der Einheit [...].»Schliemann interpretiert also die "Einheit der Kategorien" als die "Kategorie der Einheit." Es sind dies aber zwei verschiedene Begriffe von Einheit. Bei der Kategorie der Einheit handelt es sich um eine quantitative Einheit. Dage- 
gen ist die Einheit der Kategorien eine qualitative und "höhere "Einheit, die zurückzuführen ist auf die ursprüngliche synthetische Einheit der Apperzeption (Siehe B131). Eine Folge dieser falschen Identifizierung von quantitativer und qualitativer Einheit ist, dass Schliemann auf Seite 52 die Kategorie der Einheit als wesentlich für den kantischen Unterschied zwischen Erscheinung und Phänomen betrachtet, indem das Phänomen ausgelegt wird als die (wenigstens) durch die Kategorie der Einheit bestimmte Erscheinung. Auf den Seiten 58-59 wird aber dieser Ansicht von Schliemann selbst widersprochen. Dort schreibt er ganz richtig, dass auch die Erscheinung als unbestimmter Gegenstand der empirischen Anschauung schon wenigstens durch die Kategorien der Quantität bestimmt sei. In der Fußnote auf Seite 60 schreibt Schliemann dann Folgendes: »Es dürfte eine nahe liegende Hypothese sein, dass sich die Erscheinungen mithilfe der dynamischen Kategorien als Phänomene bestimmen lassen, da diese gemäß Kants Äußerungen »auf die Existenz [der ...] Gegenstände [...] gerichtet sind «(B110) «. Da Schliemann den ersten Abschnitt seines ersten Kapitels schon dem Unterschied zwischen Phänomen und `Apparensく (so nennt er die Erscheinung als unbestimmter Gegenstand) gewidmet hat (ganze 19 Seiten), hätte meines Erachtens dieser Unterschied vollständig aufgeklärt werden sollen.

Die Erscheinung (als unbestimmter Gegenstand einer empirischen Anschauung) ist nun in der Tat nicht darin unbestimmt, dass sie keine Größe wäre. Es sind andererseits aber auch nicht alle dynamische Kategorien für die Bestimmung des Gegenstandes als Phänomen verantwortlich: da die Modalkategorien keine Gegenstandsbestimmungen liefern, kommen nur noch die Kategorien der Relation in Frage. Das erste Kategorienpaar der Relation hat dabei die wichtigste Rolle, weil es den erscheinenden Gegenstand als Substanz oder Akzidenz bestimmt. In der Tat kann erst eine als Substanz oder Akzidenz bestimmte Erscheinung bei Kant ’Phänomen genannt werden. Erscheinungen des inneren Sinnes können demnach nur dann, wenn sie als Akzidenzen einer (freilich unbekannten) Substanz bestimmt werden, als Phänomenen betrachtet werden. Das heißt, dass alle Phänomene als Vorstellungen von Objekten nur Akzidenzen (meines unbekannten Selbst als Substanz) sein können. Von diesen Vorstellungen von Objekten ausgehend aber muss der Verstand klären - und dabei spielt nun das zweite Kategorienpaar der Relation die wichtigste Rolle, weil nur durch sie die als Akzidenzen bestimmte Erscheinungen als notwendige und also objektive Phänomene verstanden werden können -, ob es sich um bloße Inhalte des inneren Sinnes handelt (ob sie bloß in der Zeit sind), oder ob ihnen auch ein im Raum angesiedeltes Objekt korrespondiert. Nur in dem letzten Fall (als nicht nur in der Zeit gegeben) können die 
erscheinenden unbestimmten Gegenstände als gekannte (aber niemals als notwendig zu bezeichnende) Substanz-Phänomene bestimmt werden.

Was nun den Unterschied zwischen einem bloß Mannigfaltigen der Anschauung und der Erscheinung als (unbestimmtem) Gegenstand betrifft, schreibt Schliemann: »Das an sich zerstreute und einzelne Mannigfaltige der Empfindung muss unter den Begriff der Einheit gebracht werden, um eine Einheit der Anschauung und dadurch einen Anschauungsgegenstand zustande zu bringen." Das sunter den Begriff der Einheit bringen einer Erscheinung wird von Schliemann ausgelegt als die »Fortsetzung der produktiven Synthesis einer gewissen Art« (A170/B212). Und diese `Fortsetzung der produktiven Synthesis` ist »die reine Synthesis gemäß einer Regel der Einheit nach Begriffen überhaupt, die die Kategorie ausdrückt (A142/ B181), mithin das "Schema eines reinen Verstandesbegriffs" (ebd.). Dieses \Schema<, als quantum continuum, ist also nach Schliemann (nur) das "Schema der Kategorie der Einheit« (S. 53). Entsprechend ist dann die »Wiederholung einer immer aufhörenden Synthesis" (A170/B212) (als compositum) das Schema der Vielheit, und die »vollendete Wiederholung einer immer aufhörenden Synthesis« (als totum) das Schema der Allheit.

Nach einer Erklärung des Begriffes Subsumtion bei Kant, wonach man erwarten muss, dass die Grundsätze kategorische Allsätze sind, die Erscheinungen unter Kategorien subsumieren, gibt Schliemann auf Seite 84 eine vorläufige Formulierung der Grundsätze der Quantität:

Jede Erscheinung ist eine Einheit.

Jede Erscheinung ist eine Vielheit.

Jede Erscheinung ist eine Allheit.

Dass alle Erscheinungen zugleich sowohl eine Einheit als auch eine Vielheit und eine Allheit sein würden, findet Schliemann widersprüchlich. Die Ansicht, dass dies auch für Kant widersprüchlich gewesen sein dürfte, begründet er, indem er auf die Postulate des empirischen Denkens verweist, die dieser einfachen Struktur zufolge so lauten sollten:

Jede Erscheinung ist möglich.

Jede Erscheinung ist wirklich.

Jede Erscheinung ist notwendig.

In Bezug auf diese Formulierung schreibt Schliemann auf Seite 85: "Der Verdacht, der sich gegenüber den Quantitätskategorien erhob, dass sie nicht alle in demselben Sinne zugleich wahr sein können, bestätigt sich hinsichtlich der Grundsätze der Modalität. Zwar ist sicherlich das, was notwendig ist, auch wirklich und dieses wiederum möglich. Aber ohne Zweifel würde man doch den Bereich des Möglichen für größer halten als den des Notwendigen, so dass man nur schwer glauben mag, dass alle Erscheinungen nicht nur möglich, sonder auch notwendig sind." Danach verweist Schliemann auf den Wortlaut der Postulate, und zeigt, dass immer eine gewisse Bedingung die Gültigkeit des Kategorialsatzes ein- 
schränkt. Zum Beispiel kann das Postulat der Möglichkeit auf folgende Weise paraphrasiert werden, damit die Bedingung deutlich zu Tage tritt: "Alle Erscheinungen, die mit den formalen Bedingungen der Erfahrung übereinkommen, sind möglich." Nach Schliemanns These stimmen diese im Wortlaut aller Postulate auftretenden Bedingungen nun mit den Schemata der Modalität überein. Diese Argumentation hätte überzeugender sein können: Erstens muss man daran erinnern, dass Kant den Unterschied zwischen Grundsätzen »des mathematischen Gebrauchs" und solchen »des dynamischen Gebrauchs« dadurch erörtert, dass erstere »bloß auf die Anschauung gehen " und letztere "auf das Dasein einer Erscheinung überhaupt«. Dazu sagt Kant Folgendes: "Die Bedingungen a priori der Anschauung sind aber in Ansehung einer möglichen Erfahrung durchaus notwendig, die des Daseins der Objekte einer möglichen empirischen Anschauung an sich nur zufällig."(A160/B199) Wenn nun die Kategorien der Quantität (Kant nennt sie auch Kategorien der Größe) diejenigen apriorischen Begriffe sind, die notwendig dafür sind, dass ein Mannigfaltiges der Anschauung als eine Größe angeschaut werden kann, ist es keineswegs auszuschließen, dass jede (wenigstens als Gegenstand bestimmte) Erscheinung zugleich Einheit, Vielheit und Allheit ist. Zweitens scheint Schliemann hier eine nicht-kantische Auffassung der Modalitätskategorien zu vertreten. Kant selbst warnt in Bezug auf die Modalitätskategorien vor der "Armseligkeit unserer gewöhnlichen Schlüsse, wodurch wir ein großes Reich der Möglichkeit herausbringen, davon alles Wirkliche (aller Gegenstand der Erfahrung) nur ein kleiner Teil sei.» (A231/B283) Nach Kant ist es nämlich tatsächlich so, dass jede als Akzidenz bestimmte Erscheinung sowohl möglich und (als Phänomen) wirklich als auch (hypothetisch) notwendig ist (Siehe A227/B280ff.), und die Menge des Möglichen ist bei ihm nicht größer als die Menge des Wirklichen. Es müsste also doch das Statut dieser in den Postulaten genannten Bedingungen weiter untersucht werden, bevor man daraus schließen kann, dass nicht jede als Gegenstand bestimmte Erscheinung zugleich Einheit, Vielheit und Allheit sein kann.

Seine Hauptthese (»dass die transzendentalen Schemata der Kategorien diejenigen Bedingungen sind, durch welche der Subjektbegriff der Grundsätze des reinen Verstandes auf eine bestimmte Klasse von Erscheinungen eingeschränkt wird" (87)) bestätigt Schliemann in der Folge dann aber nicht dadurch, dass er tatsächlich zeigt, wie die in den Postulaten genannten Bedingungen als Schemata der Modalität zu verstehen seien, sondern anhand einer Analyse der Analogien der Erfahrung. In Fußnote 30 heißt es dazu: »Die These, dass die in den Postulaten gebrauchten einschränkenden Bedingungen [...] die Schemata der Modalität bedeuten, möchte ich ebenfalls vertreten, halte aber den entsprechenden Nachweis für etwas umständlicher als den für die Analogien, weshalb ich mich an dieser Stel- 
le für deren Analyse entschieden habe«. Es ist nun in der Tat nicht sofort einsichtig, wie zum Beispiel die im Postulat der Möglichkeit genannte Bedingung ("mit den formalen Bedingungen der Erfahrung übereinkommen«) als das Schema der Möglichkeit (»die Zusammenstimmung der Synthesis verschiedener Vorstellungen mit den Bedingungen der Zeit überhaupt, [...], also die Bestimmung der Vorstellung eines Dinges zu irgend einer Zeit" - A144/B184) zu verstehen sei. Dies hätte Schliemann noch erklären sollen, damit seine These plausibler erscheinen würde (was Schliemann selbst auf Seite 138 auch einigermaßen zugibt, in dem letzten Abschnitt des Buches: ‘Ausblick $`$ ).

Nach der angekündigten Analyse, in der Schliemann zeigt, wie die Analogien der Erfahrung die von ihm herausgearbeitete allgemeine Struktur der Grundsätze respektieren - eine gelungene Analyse, die sicher zu den interessantesten im Buch gehört - kann er nun den Wortlaut der rekonstruierten Axiome der Anschauung preisgeben (95):

1. Jede Erscheinung, deren Anschauung durch die Fortsetzung der produktiven Synthesis einer gewissen Art erzeugt wird, ist ein Quantum (continuum), d. h. eine Einheit.

2. Jede Erscheinung, deren Anschauung durch Wiederholung einer immer aufhörenden Synthesis erzeugt wird, ist ein Kompositum, d. h. eine Vielheit.

3. Jede Erscheinung, deren Anschauung durch die vollendete Wiederholung einer immer aufhörenden Synthesis erzeugt wird, ist ein Totum, d.h. eine Allheit.

Was hierbei befremden muss ist, dass dieser von Schliemann vorgeschlagene Wortlaut der einzelnen Axiome nicht die von ihm selbst herausgestellte Struktur eines Grundsatzes überhaupt respektiert: er setzt nämlich (mittels den jeweiligen »d.h.") die Kurzformel der von ihm rekonstruierten Schemata der Kategorien der Quantität einfach mit diesen Kategorien gleich. Dass dieser Fehler geschehen konnte, wird beim Leser doch die Frage aufwerfen, ob der Rekonstruktionsversuch das Verständnis der Grundsätze wirklich weiter gebracht hat. Zur Verteidigung Schliemanns sollte man aber anführen, dass man ziemlich einfach den mit seiner Hauptthese konsistenten Wortlaut der Axiome herstellen kann. Sie müssten dann nämlich so formuliert werden:

1. Jede Erscheinung, deren Anschauung ein Quantum (continuum) ist (d.h. durch die Fortsetzung der produktiven Synthesis einer gewissen Art erzeugt wurde), ist eine Einheit.

2. Jede Erscheinung, deren Anschauung ein Kompositum ist (d.h. durch Wiederholung einer immer aufhörenden Synthesis erzeugt wurde), ist eine Vielheit.

3. Jede Erscheinung, deren Anschauung ein Totum ist (d. h. durch die vollendete Wiederholung einer immer aufhörenden Synthesis erzeugt wurde), ist eine Allheit.

Dass nun auch diese von mir umformulierten Axiome vielleicht eine gewisse Trivialität (im Sinne einer banalen Evidenz) aufweisen, darf man nicht sofort als Abwertung von Schliemanns Ergebnis gelten lassen, da gerade eine solche Selbstverständlichkeit der Axiome einen guten Grund dafür abgeben könnte, dass Kant 
versäumt hat, sie explizit zu formulieren. Das sagt auch Schliemann: „Warum hat Kant entgegen seiner Forderung die Axiome der Anschauung nicht im Einzelnen deduziert? Die Vermutung liegt nahe, dass dieses Versäumnis mit der [...] intuitiven Gewissheit der mathematisch genannten Verstandesgrundsätze zusammenhängt. [...] Anscheinend hält Kant die Axiome der Anschauung sogar für so selbstverständlich, dass er sie nicht einmal ausdrücklich nennt [...].«(S.116) Trotzdem bleibt es ungeklärt (und davon zeugt nun auch gerade der Fehler in Schliemanns Formulierung der rekonstruierten Axiome), inwiefern vor allem die

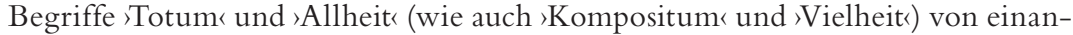
der unterschieden werden müssen.

Die Aufgabe, die Schliemann sich in dieser Arbeit gestellt hat, macht den Leser neugierig, weil es in der Tat nicht ohne weiteres klar ist, welche Sätze Kant mit den Axiomen der Anschauung vor Augen gehabt hat. Diese Aufgabe birgt aber, gerade dadurch, dass Kant nirgendwo einzelne Axiome der Anschauung als philosophische Grundsätze aufstellt, das Risiko in sich, dass das Ergebnis einer solchen Untersuchung unbefriedigend bleiben muss, zum Beispiel weil es als trivial oder als zu spekulativ betrachtet wird. Schliemann geht meistens sehr sorgfältig mit den kantischen Texten um, und von einem ungezügelten spekulativen Trieb kann bei ihm sicherlich nicht die Rede sein. In diesem Sinne gehörte es sich nicht, davon abzuraten, das Buch zur Hand zu nehmen, wenn man eine Interpretation dessen sucht, was Kant in den ersten Abschnitten des Grundsatzkapitels sagt. Die von ihm unternommene Rekonstruktion der Axiome der Anschauung (auch in der von mir umformulieren Fassung) bringt aber meines Erachtens das Verständnis dieser Abschnitte der Kritik nun leider auch nicht wesentlich weiter.

Henny Blomme

School of Philosophy, Psychology and Language Sciences

The University of Edinburgh

Dugald Stewart Building

3 Charles Street

Edinburgh EH8 9AD

hennyblomme@gmail.com 